\section{Architektur des Corpus spongiosum}

Ottenhof SR et al. Architecture of the Corpus Spongiosum: An Anatomical Study. J Urol 2016; 196: 919-925

Rekonstruktive Eingriffe an der männlichen Urethra können nicht nur bei Urethrastrikturen, sondern auch bei angeborenen Fehlbildungen wie ausgedehnten Hypospadien notwendig werden. Oft sind dafür Schleimhautgrafts notwendig, um eine spannungsfreie Anastomose zu erreichen, aber hier kann die Blutversorgung zum limitierenden Faktor werden. Mediziner aus den Niederlanden haben daher eine detaillierte anatomische Studie angestellt.

Sarah Ottenhof und ihre Kollegen gingen von der Annahme aus, dass für eine optimale Blutversorgung der mit Schleimhauttransplantaten rekonstruierten Urethra ein funktionelles Corpus spongiosum notwendig ist. Eine akkurate Wiederherstellung des die Urethra umgebenden Schwellkörpers könnte demnach die Ergebnisse der Urethroplastik verbessern. Dazu ist aber eine genaue Kenntnis der Anatomie notwendig, und die haben die Wissenschaftler nun untersucht. Dazu haben sie den Penis von 2 verstorbenen Männern (59 und 91 Jahre alt) entnommen und das Corpus spongiosum und die beiden Corpora cavernosa in Gänze erhalten. Bei einem Penis wurde mit physiologischer Kochsalzlösung eine Erektion erzeugt. Anschließend wurden 6 Gefrierschnitte in der Transversalebene und 7 Schnitte in der Frontalebene auf Höhe des Corpus spongiosum angefertigt. Die Präparate wurden dann in modifizierter Mallory-Cason-Lösung angefärbt, auf Filterpapier aufgespannt, fotografiert und digital rekonstruiert. In diesen Aufnahmen vermaßen die Forscher dann eine Reihe anatomischer Parameter. In den transversalen Schnitten betrug die mittlere relative Oberfläche der Gefäßsinus in den verschiedenen Präparaten im Mittel

- $59,9 \%$ im schlaffen Penis und

- $76,6 \%$ im erigierten Penis
In der Frontalebne lagen diese Werte bei

- $52,8 \%$ bzw.

- $73,9 \%$

Daraus errechnete sich eine relative Zunahme des Volumens um 29\% im Querschnitt bzw. 40 \% im Längsschnitt während der Erektion. Die Schwellkörperhohlräume zeigten sich dabei in den Frontalschnitten ausgedehnter und weniger stark septiert als in den Transversalschnitten. Insgesamt unterschieden sich die verschiedenen Schnitte mit Ausnahme der Glans penis nicht wesentlich in ihrem Aufbau.

\section{FAZIT}

Ottenhof und Kollegen sind der Meinung, dass diese Erkenntnisse dazu beitragen können, bei rekonstruktiven Eingriffen an der Urethra die Operation im Voraus zu planen. Dabei wäre auch der 3-dimensionale Druck eines strukturellen Gerüsts für das Corpus spongiosum im Rahmen eines Tissue Engineering denkbar. Einschränkend gilt, dass nur 2 Organe untersucht wurden, für die zwar das Alter der Männer bekannt war, nicht aber deren hormoneller Status, der die Ergebnisse beeinflussen könnte.

\section{Dr. Elke Ruchalla, Bad Dürrheim}

\section{Kommentar}

Ottenhof et al. von der Universitätsklinik Utrecht untersuchten die anatomischen Gegebenheiten des Corpus spongiosum im Rahmen einer Kadaverstudie. Die beiden Kadaver stammten von einem 59 und einem 91 jährigen Mann. Hierzu wurden ein nicht erigierter Penis und ein durch Kochsalzlösung artifiziell erigierter Penis eingefroren und dann zur weiteren Untersuchung alle $2 \mathrm{~cm}$ Schnittflächen zur Untersuchung angefertigt. Nach Färbung der Schnittflächen wurden diese digitalisiert und eine 3D-Rekonstruktion durchgeführt.

Die Vorliegende Arbeit beschäftigt sich erstmals ausdrücklich mit der Veränderung des Corpus spongiosum während der Erek- tion gegenüber dem nicht erigierten Penis. Dies ist begrüßenswert, da sich durch eine genauere Untersuchung dieser Veränderungen - wie auch des Corpus spongiosum überhaupt - wesentliche Erkenntnisse für ein tissue engeneering bei Harnröhrenrekonstruktionen und des Corpus spongiosum gewinnen lassen. Darüber hinaus hat es Bedeutung für Techniken der Harnröhrenrekonstruktion. In der Therapie der Harnröhrenstriktur stellt das Corpus spongiosum einen wichtigen Faktor zur Deckung von Mundschleimhauttransplantaten dar, wodurch eine erfolgreiche Rekonstruktion erst möglich wird. Besonders deutlich wird die Bedeutung des Corpus spongiosum in einer prospektiven Studie von Bhat et al. bei 113 Patienten mit Hypospadie. Hier zeigte sich eine Erhöhung des Risikos zur Ausbildung einer Harnröhrenfistel nach Rekonstruktion von 11,3 \% bei Patienten mit gut vaskularisiertem Spongiosagewebe auf 23,03\% bei Patienten, deren Corpus spongiosum nur dünn und fibrotisch war [1].

Die Hauptaussage der Studie von Ottenhof et al. ist, dass sich im Transversalschnitt eine Zunahme im vaskulären Lumen von $60 \%$ im nicht erigierten Penis auf $77 \%$ im erigierten Penis und im Frontalschnitt von 53 auf $74 \%$ zeigte. Im Frontalschnitt zeigte sich hierbei eine abnehmende vaskuläre Oberfläche im Bereich der Glans bei gleichzeitiger Zunahme von Bindegewebe.

Die Hauptlimitation der Studie besteht in der Zahl von nur 2 untersuchten Probanden - mithin einer pro Gruppe -, die noch dazu eine Altersdifferenz von 32 Jahren aufweisen. Inwieweit also die beiden untersuchten Penisse miteinander vergleichbar sind und ob die Untersuchungsergebnisse des Corpus spongiosum eines 91-jährigen auf deutlich jüngere Patienten übertragbar sind bleibt offen.

\section{Schlussfolgerung}

Letztlich ist die Studie als „proof of principle“ der verwendeten Untersuchungstechnik sehr interessant, die übrigen Ergebnisse bedürfen allerdings der Validierung an einer größeren Probandenzahl und sollte zumindest in alters-gleichen Gruppen erfolgen. 
Der Autor

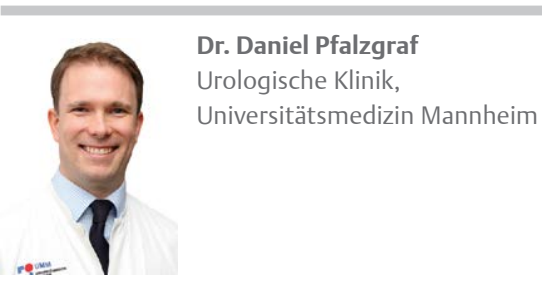

Literatur

[1] Bhat A, Sabharwal K, Bhat M et al. Outcome of tubularized incised plate urethroplasty with spongioplasty alone as additional tissue cover: A prospective study. Indian J Urol 2014; 30: 392-397 